\title{
Computational Mechanics: Natural Computation and Self-organization
}

\author{
James P. Crutchfield \\ Complexity Sciences Center \\ Physics Department \\ University of California at Davis \\ Davis, California 95616 USA \\ http://cse.ucdavis.edu/ chaos/
}

\begin{abstract}
The tutorial explores how nature's structure reflects how nature computes. It reviews intrinsic unpredictability (deterministic chaos) and the emergence of structure (self-organization) in natural complex systems. Using statistical mechanics, information theory, and computation theory, it develops a systematic framework for analyzing processes in terms of their causal architecture. This is determined by answering three questions: (i) How much historical information does a process store? (ii) In what architecture is that information stored? And (iii) how is the stored information used to produce future behavior? The answers to these questions tell one how a system intrinsically computes.
\end{abstract}

Readings. For the tutorial, see the articles, going from less to more technical (that is, the recommended reading order):

1. Is Anything Ever New? Considering Emergence http://cse.ucdavis.edu/ cmg/compmech/pubs/EverNewTitlePage.htm

2. Regularities Unseen, Randomness Observed: Levels of Entropy Convergence http://cse.ucdavis.edu/ cmg/compmech/pubs/ruro.htm

3. The Calculi of Emergence: Computation, Dynamics, and Induction http://cse.ucdavis.edu/ cmg/compmech/pubs/ CalcEmergTitlePage.htm

4. Computational Mechanics: Pattern and Prediction, Structure and Simplicity http://cse.ucdavis.edu/ cmg/compmech/pubs/cmppss.htm

5. The Organization of Intrinsic Computation: Complexity-Entropy Diagrams and the Diversity of Natural Information Processing http://cse.ucdavis.edu/ cmg/compmech/pubs/oic.htm

6. Structure or Noise?

http://cse.ucdavis.edu/ cmg/compmech/pubs/son.htm 